\title{
HLA Match
}

National Cancer Institute

\section{Source}

National Cancer Institute. HLA Match. NCI Thesaurus. Code C129972.

The state when a recipient and their donor have the same alleles for tissue markers. A full match includes HLA-A, HLA-B, HLA-C, and HLA-DRB1. HLA match typing may also include alleles in the HLA-DQ serogroup. 